Article

\title{
Cosmological Constraints on the Coupling Model from Observational Hubble Parameter and Baryon Acoustic Oscillation Measurements
}

\author{
Shulei Cao ${ }^{1,2} \mathbb{D}$, Tong-Jie Zhang ${ }^{1,3, * \mathbb{D}}$, Xinya Wang ${ }^{4}$ and Tingting Zhang ${ }^{5}$ \\ 1 Department of Astronomy, Beijing Normal University, Beijing 100875, China; shulei@phys.ksu.edu \\ 2 Department of Physics, Kansas State University, 116 Cardwell Hall, Manhattan, KS 66502, USA \\ 3 Institute for Astronomical Science, Dezhou University, Dezhou 253023, China \\ 4 Department of Industrial and Manufacturing Systems Engineering, Kansas State University, \\ Manhattan, KS 66506, USA; lawrence15@ksu.edu \\ 5 PLA Army Engineering University, Nanjing 210017, China; 101101964@seu.edu.cn \\ * Correspondence: tjzhang@bnu.edu.cn
}

Citation: Cao, S.; Zhang, T.-J.;

Wang, X.; Zhang, T. Cosmological

Constraints on the Coupling Model from Observational Hubble

Parameter and Baryon Acoustic

Oscillation Measurements. Universe

2021, 7, 57. https://doi.org/10.3390/

universe7030057

Academic Editor: Pedro Avelino

Received: 29 January 2021

Accepted: 2 March 2021

Published: 5 March 2021

Publisher's Note: MDPI stays neutral with regard to jurisdictional claims in published maps and institutional affiliations.

Copyright: (c) 2021 by the authors. Licensee MDPI, Basel, Switzerland. This article is an open access article distributed under the terms and conditions of the Creative Commons Attribution (CC BY) license (https:/ / creativecommons.org/licenses/by/ $4.0 /)$.

\begin{abstract}
In the paper, we consider two models in which dark energy is coupled with either dust matter or dark matter, and discuss the conditions that allow more time for structure formation to take place at high redshifts. These models are expected to have a larger age of the universe than that of $\Lambda \mathrm{CDM}$ [universe consists of cold dark matter (CDM) and dark energy (a cosmological constant, $\Lambda$ )], so it can explain the formation of high redshift gravitationally bound systems which the $\Lambda C D M$ model cannot interpret. We use the observational Hubble parameter data (OHD) and Hubble parameter obtained from cosmic chronometers method $(H(z))$ in combination with baryon acoustic oscillation (BAO) data to constrain these models. With the best-fitting parameters, we discuss how the age, the deceleration parameter, and the energy density parameters evolve in the new universes, and compare them with that of $\Lambda \mathrm{CDM}$.
\end{abstract}

Keywords: dark energy; cosmological parameters; cosmology: observations

\section{Introduction}

The observational developments recently have provided cosmology with a standard model, which describes the universe by the Friedmann-Lemaitre-Robertson-Walker (FLRW) metric, with cold pressureless matter contributing roughly $1 / 3$ and the negative pressure dark energy (DE) contributing the remaining $2 / 3$ of the critical energy density [1-3].

Agreeing with most observations, $\Lambda \mathrm{CDM}$ [universe consists of cold dark matter (CDM) and dark energy (a cosmological constant, $\Lambda$ )] [4] is accepted by most of the scientists in cosmology. However, recent observations provide some conflicts with $\Lambda \mathrm{CDM}$. For example, over 400 quasars (QSOs) with $z>4$ are found, and the seven highest redshift quasars have $z>5.7[5,6]$. If their centers are black holes, these black holes must have a scale of $10^{9} \mathrm{M}_{\odot}$. Whether such supermassive black holes can form in the $\Lambda$ CDM universe with an age less than $10^{9}$ years at $z \sim 6$ [7] remains an open question. Moreover, as stated in [8], the recent discovery of SDSS 010013.02+280225.8 (hereinafter "J0100+2802"), an ultraluminous quasar at redshift $z=6.30$, has aggravated the problem of supermassive black-hole growth and evolution in the early Universe [9]. Not to mention the issues that the 14-Gyr age of the universe obtained by Pont et al. [10], which is in tension with $13.797 \pm 0.023 \mathrm{Gyr}$ [11], the 3.5-Gyr-old radio galaxy 53W091 at $z=1.55$ and 4-Gyr-old radio galaxy 53 W069 [12,13]. In addition, the existence of Pop III stars $(z \geq 17)$ which may have been responsible for ionizing the universe at lower redshifts is also problematic in the $\Lambda$ CDM [5].

Motivated by the above issues and the works of Hao Wei [14-16], here we intend to test a model of dark energy (DE) coupled with matter that ameliorates the aging problem. 
The coupling between DE and dust matter has been discussed in detail [17-19]. In the literature, the coupling between $\mathrm{DE}$ and dark matter (DM) has been investigated to explain the Hubble constant $\left(H_{0}\right)$ and $\sigma_{8}$ tensions [20-26], where $\sigma_{8}$ measures the amplitude of the (linear) power spectrum on the scale of $8 \mathrm{~h}^{-1} \mathrm{Mpc}$, with $h=H_{0} /\left(100 \mathrm{~km} \mathrm{~s}^{-1} \mathrm{Mpc}^{-1}\right)$ being the reduced Hubble constant. It would be interesting to also consider a model with $\mathrm{DE}$ and DM coupling. Therefore, for comparison purpose, we select three coupling forms of these two models and use observational Hubble parameter data (OHD) and Hubble parameter obtained from cosmic chronometers method (denoted as $H(z)$ ) in combination with baryon acoustic oscillation (BAO) data to constrain the cosmological and nuisance parameters in the given models. Finally, we explore the general properties of the best-fitting models and their implications, and determine the most favored model.

This paper is organized as follows: In Section 2, we briefly review the standard cosmology and its challenges. In Section 3, we describe the coupling models in details. The constraints on the models are presented in Section 4. In Section 5, we discuss the properties of the best-fitting models and their merits. Finally, the conclusions and discussions are given in Section 6.

\section{The Standard Cosmology and Its Challenges}

The current standard cosmology is mainly based on the Einstein's general relativity, and FLRW metric, where the homogeneous and isotropic solution of Einstein's field equations, is given by

$$
d s^{2}=-d t^{2}+a^{2}(t)\left[\frac{d r^{2}}{1-k r^{2}}+r^{2} d \theta^{2}+r^{2} \sin ^{2} \theta d \phi\right] .
$$

$a(t)$ is the scale factor, $r, \theta$ and $\phi$ are dimensionless comoving coordinates, and $k=0, \pm 1$ represent the curvature of the spatial Section [27]. The homogeneous matter in the universe behaves as a tensor

$$
T^{\mu v}=(\rho+P) U^{\mu} U^{v}+P g^{\mu \nu},
$$

where $\rho$ and $P$ represent the density and the pressure of the matter, and $U^{\mu}$ and $g_{\mu \nu}$ denote the 4-velocity of the matter and space-time metric, respectively. When $U=(1,0,0,0)$, matter is static in the reference coordinate, but comoving with the expanding universe. From Friedmann equations, one can get the the expression of Hubble parameter $H(z)$ in flat $\Lambda \mathrm{CDM}$,

$$
H^{2}=H_{0}^{2}\left(\Omega_{m_{0}}(1+z)^{3}+\Omega_{r_{0}}(1+z)^{3}+\Omega_{\Lambda}\right),
$$

where $\Omega_{m_{0}}, \Omega_{r_{0}}$, and $\Omega_{\Lambda}$ are current radiation energy, current dust matter, and constant DE density parameters, respectively, and subscript " 0 " denotes the present value hereafter. Note that $\Omega_{r_{0}}$ is accurately measured, being $\Omega_{r_{0}}=2.47 \times 10^{-5} h^{-2}$ [28], and the current neutrino energy density parameter $\Omega_{v_{0}}$ is given by

$$
\Omega_{v_{0}}=\frac{\sum m_{v}}{93.14 h^{2}},
$$

where $\sum m_{v}=0.06 \mathrm{eV}$ is the total neutrino mass and the effective number of massless neutrino species $N_{\text {eff }}=3.045$ [28]. Though $\Lambda$ CDM is generally accepted by most scientists and agreed mostly with the observations, it appears that the recent observations at modest redshifts $(6 \leq z \leq 20)$ have some surprises in store for $\Lambda$ CDM [5].

In fact, over 400 QSOs with redshifts $z>4$ are known at present, and the seven highest redshift quasars have $z>5.7$. If quasars shine by virtue of an accreting black hole at their centers, then all these QSOs must host $\geq 10^{9} \mathrm{M}_{\odot}$ black holes [7], and it takes at least $7 \times 10^{8}$ years for such a black hole to form. We define the age of the universe at the redshifts $z, t_{z}$, as

$$
t_{z}=\int_{z}^{\infty} \frac{d z}{(1+z) H(z)}
$$


Considering $H_{0}=67.4 \mathrm{~km} \mathrm{~s}^{-1} \mathrm{Mpc}^{-1}$ and $\Omega_{m_{0}}=0.315$ [11], and substituting Equation (3) into Equation (5), we get the age of universe at $z \sim 6$ as $9.3 \times 10^{8}$ years which is merely enough for the formation of $10^{9} M_{\odot}$ black holes.

The following arguments are summarized by Melia and McClintock [8]. In the standard context, the universe became transparent about 0.4 Myr after the big bang, descending into the so-called Dark Ages, which ended several hundred million years later. After that, density perturbations condensed into stars and early galaxies, producing ionizing radiation and the epoch of re-ionization (EoR) began. Standard astrophysical principles suggest that ionizing radiation was produced by Pop II and III stars. The EoR was constrained by Zaroubi et al. [29] at $6 \lesssim z \lesssim 15$. How the universe evolved through the Dark Ages and into the EoR was studied by many detailed simulations (e.g., [30]), which show that primordial gas clouds formed in dark-matter halos with virial temperature $1000 \mathrm{~K}$ and mass $10^{6} \mathrm{M}_{\odot}$ (so-called "minihaloes"; $M_{\odot}$, solar mass). In the standard CDM model, the minihaloes that were the first sites for star formation are expected to be in place at redshift $z \approx 20$, when the age of the universe was just a few hundred million years [31]. After at least $100 \mathrm{Myr}$, 5-20 $M_{\odot}$ black-hole seeds were created, presumably following the supernova explosion of evolved Pop II (and possibly Pop III) stars. Conventionally, the black-hole mass as a function of time can be expressed by Salpeter relation (see [8] and references therein for more details)

$$
M(t)=M_{\text {seed }} \exp \frac{t-t_{\text {seed }}}{45 \mathrm{Myr}},
$$

where $M_{\text {seed }}$ is the black-hole seed mass produced at time $t_{\text {seed }}$. Note that $M(t)$, being a model-dependent quantity, may present dependence on cosmological parameters, especially on $H_{0}$ [32]. Assuming $M_{\text {seed }}=20 M_{\odot}$ (upper bound), the minimal growth time from the Salpeter relation is $t-t_{\text {seed }} \sim 910 \mathrm{Myr}$ for an inferred mass of approximately 10-12 $\times 10^{9} M_{\odot}$. Since in $\Lambda \mathrm{CDM}$ (with $H_{0}=67.4 \mathrm{~km} \mathrm{~s}^{-1} \mathrm{Mpc}^{-1}$ and $\Omega_{m_{0}}=0.315$ given by [11] $), t(z=6.3) \sim 873 \mathrm{Myr}$, not only is this age of quasar J0100+2802 inconsistent with the transition from the Dark Ages to the EoR, but also the quasar would have had to grow beyond the big bang. This is still true even if we are using the lower bounds of $H_{0}=66.82 \mathrm{~km} \mathrm{~s}^{-1} \mathrm{Mpc}^{-1}$ and $\Omega_{m_{0}}=0.308$ from [11], which result in $t(z=6.3) \sim 891$ Myr.

In order to improve these problems, we aim to test a new model to agree better with the observations. Indeed, a smaller value of $H(z)$ at high redshifts can result in a larger age of the universe, so can an oscillating $H(z)$. Note that there also exist other challenges, such as $H_{0}$ and $\sigma_{8}$ tensions, but here we mainly focus on the aging problems. We will introduce the coupling model in the following section.

\section{Coupling between Dark Energy and Dust/Dark Matter}

\subsection{Dark Energy Coupled with Dust Matter}

The standard theory of $\Lambda \mathrm{CDM}$ is based on the assumption that all the cosmological compositions evolve independently, while first we assume that the dust matter and DE exchange energy through interaction according to

$$
\dot{\rho}_{\mathrm{X}}+3 H\left(1+w_{\mathrm{X}}\right) \rho_{\mathrm{X}}=-3 \lambda H \rho_{m}
$$

and

$$
\dot{\rho}_{m}+3 H \rho_{m}=3 \lambda H \rho_{m},
$$

and the conservation equation for the radiation energy density is

$$
\dot{\rho}_{r}+4 H \rho_{r}=0,
$$

which preserves the total energy conservation equation $\dot{\rho}_{\text {tot }}+3 H\left(\rho_{\text {tot }}+P_{\text {tot }}\right)=0$ [14-16], where $\lambda, \rho_{m}, \rho_{\mathrm{X}}, \rho_{r}$, and $\dot{\rho}$ denote the coupling coefficient, the matter density, the DE density, the radiation energy density, and their time derivatives. Some researchers use 
this model to discuss the Chameleon DE and its test of the solar system [17-19]. Here, $\lambda$ determines the coupling form between the matter and DE, and the DE equation of state (EoS) $w_{\mathrm{X}}=P_{\mathrm{X}} / \rho_{\mathrm{X}}$ is constant. Here we only consider the spatially-flat FLRW universe. With $3 \lambda H \rho_{m}$ as the interaction term, here we assume that the coupling coefficient takes the form $\lambda=A_{1}^{3} \cos \left[\frac{\ln (1+z)}{A_{2}}-A_{1} \pi\right]$ [14], in which $A_{1}$ and $A_{2}$ are undetermined constant coefficients. Substituting the expression of $\lambda$ into Equation (8), we can get the expression of $\rho_{m}$,

$$
\rho_{m}(z)=\rho_{m_{0}}(1+z)^{3} \exp \left\{-3 A_{1}^{3} A_{2}\left[\sin \left(A_{1} \pi\right)+\sin \left[\frac{\ln (1+z)}{A_{2}}-A_{1} \pi\right]\right]\right\},
$$

where $\rho_{m_{0}} \equiv \rho_{m}(z=0)$. Substituting Equation (10) into Equation (7), we can obtain the expression of $\rho_{\mathrm{X}}$,

$$
\rho_{\mathrm{X}}(z)=(1+z)^{3\left(1+w_{\mathrm{X}}\right)}\left[\rho_{\mathrm{X}_{0}}+\rho_{m_{0}} I(z)\right]
$$

where

$$
\begin{aligned}
I(z)= & \int_{0}^{z} \frac{3 A_{1}^{3}}{(1+x)^{3 w_{X}+1}} \cos \left[\frac{\ln (1+x)}{A_{2}}-A_{1} \pi\right] \\
& \exp \left\{-3 A_{1}^{3} A_{2}\left[\sin \left[\frac{\ln (1+x)}{A_{2}}-A_{1} \pi\right]+\sin \left(\frac{\pi}{A_{1}}\right)\right]\right\} d x .
\end{aligned}
$$

Similarly, from Equation (9), we can get

$$
\rho_{r}(z)=\rho_{r_{0}}(1+z)^{4} .
$$

Therefore in this model, we have

$$
\frac{\rho_{m}+\rho_{\mathrm{X}}+\rho_{r}}{\rho_{\text {crit }}}=\Omega_{m}(z)+\Omega_{\mathrm{X}}(z)+\Omega_{r}(z)=1,
$$

in which $\Omega_{m}=\rho_{m} / \rho_{\text {crit }}, \Omega_{\mathrm{X}}=\rho_{\mathrm{X}} / \rho_{\text {crit }}$, and $\Omega_{r}=\rho_{r} / \rho_{\text {crit }}$ are the dust matter density, DE density, and radiation energy density parameters, respectively, with $\rho_{\text {crit }} \equiv 3 H^{2} / 8 \pi G$ being the critical density. Furthermore, $\Omega_{m_{0}}+\Omega_{\mathrm{X}_{0}}+\Omega_{r_{0}}=1$ is satisfied. Then, the Friedmann equation becomes

$$
\begin{aligned}
\frac{H^{2}}{H_{0}^{2}}= & \Omega_{m_{0}}(1+z)^{3} \exp \left\{-3 A_{1}^{3} A_{2}\left[\sin \left(A_{1} \pi\right)+\sin \left[\frac{\ln (1+z)}{A_{2}}-A_{1} \pi\right]\right]\right\} \\
& +(1+z)^{3\left(1+w_{\mathrm{x}}\right)}\left\{1-\Omega_{\mathrm{m}_{0}}-\Omega_{r_{0}}+\Omega_{m_{0}} I(z)\right\}+\Omega_{r_{0}}(1+z)^{4}
\end{aligned}
$$

This equation is the $H(z)$ expression of this coupling model (denoted as "Model 1" hereinafter).

We could also simply put $\lambda=$ const. In this case (denoted as "Model 2" hereinafter), the Hubble parameter becomes

$$
H(z)=H_{0} \sqrt{\frac{w_{\mathrm{X}} \Omega_{m_{0}}}{\lambda+w_{\mathrm{X}}}(1+z)^{3(1-\lambda)}+\left(1-\Omega_{r_{0}}-\frac{w_{\mathrm{X}} \Omega_{m_{0}}}{\lambda+w_{\mathrm{X}}}\right)(1+z)^{3\left(1+w_{\mathrm{X}}\right)}+\Omega_{r_{0}}(1+z)^{4}} .
$$

\subsection{Dark Energy Coupled with Dark Matter}

If we consider the coupling between DE and (cold) DM (see [33] for review), the interaction equations read

$$
\dot{\rho}_{\mathrm{X}}+3 H\left(1+w_{\mathrm{X}}\right) \rho_{\mathrm{X}}=-3 \lambda H \rho_{X},
$$

and

$$
\dot{\rho}_{c}+3 H \rho_{c}=3 \lambda H \rho_{X}
$$


where $\rho_{c}$ is the energy density for cold DM. Here we also consider $\lambda=$ const. Consequently, we can obtain the expressions of $\rho_{\mathrm{X}}$

$$
\rho_{\mathrm{X}}(z)=\rho_{\mathrm{X}_{0}}(1+z)^{3\left(1+w_{\mathrm{X}}+\lambda\right)},
$$

and $\rho_{c}$

$$
\rho_{c}(z)=(1+z)^{3}\left\{\rho_{c_{0}}+\frac{\lambda}{w_{\mathrm{X}}+\lambda} \rho_{\mathrm{X}_{0}}\left[(1+z)^{3\left(w_{\mathrm{X}}+\lambda\right)}-1\right]\right\},
$$

where $\rho_{X_{0}} \equiv \rho_{X}(z=0)$ and $\rho_{c_{0}} \equiv \rho_{c}(z=0)$. The conservation equation for the energy density of the barynoic matter is

$$
\dot{\rho}_{b}+3 H \rho_{b}=0,
$$

which leads to

$$
\rho_{b}(z)=\rho_{b_{0}}(1+z)^{3} .
$$

In this case (denoted as "Model 3" hereinafter), the dust matter density becomes $\rho_{m}=\rho_{b}+\rho_{c}$. Following the same definitions of the cosmological density parameters as above, $\Omega_{b}, \Omega_{c}$, and $\Omega_{\mathrm{X}}$ are the baryon, DM, and DE density parameters, respectively, and $\Omega_{b}+\Omega_{c}+\Omega_{X}+\Omega_{r}=1$. The Friedman equation then becomes

$$
H(z)=H_{0} \sqrt{(1+z)^{3}\left\{\Omega_{b_{0}}+\Omega_{c_{0}}+\frac{\Omega_{X_{0}}}{w_{\mathrm{X}}+\lambda}\left[\left(w_{\mathrm{X}}+2 \lambda\right)(1+z)^{3\left(w_{\mathrm{X}}+\lambda\right)}-\lambda\right]+\Omega_{r_{0}}(1+z)\right\}},
$$

where $\Omega_{m_{0}}=\Omega_{b_{0}}+\Omega_{c_{0}}$ and $\Omega_{m_{0}}+\Omega_{\mathrm{X}_{0}}+\Omega_{r_{0}}=1$.

In the following section, we will constrain these models with OHD and $H(z)+$ BAO combination.

\section{Constraints on the Coupling Model with Observational Data}

The 40 OHD we used here are based on cosmic chronometers (see [34-38] for the usage of these $31 \mathrm{H}(z)$ data) and radial BAO size methods, as shown in Table 1, where we could use them together is because they are statistically-independent (systematic uncertainty of individual data point is accounted for, and in fact, based on the reduced $\chi^{2}$ value listed in Table 2, the errors are overestimated). Systematic errors that affect $H(z)$ measurements from cosmic chronometers were studied [39,40], and were recently re-examined in [41,42]. Note that the statistically-dependent (correlated) data [43] has been removed (which is included in BAO measurements) compared with what used in [44] (see also $[45,46]$ for more details regarding $\mathrm{OHD}$ ).

The 11 BAO data used here are the same as what used in Cao et al. (see [38] for more details). The systematic uncertainties of these BAO data are examined and found to be either negligible compared with the statistical uncertainties or included in the corresponding covariance matrix. 
Table 1. The current available OHD dataset.

\begin{tabular}{|c|c|c|c|}
\hline$z$ & $H(z)^{a}$ & Method $^{b}$ & Ref. \\
\hline 0.0708 & $69.0 \pm 19.68$ & I & Zhang et al. (2014)-[47] \\
\hline 0.09 & $69.0 \pm 12.0$ & I & Jimenez et al. (2003)-[48] \\
\hline 0.12 & $68.6 \pm 26.2$ & I & Zhang et al. (2014)-[47] \\
\hline 0.17 & $83.0 \pm 8.0$ & I & Simon et al. (2005)-[49] \\
\hline 0.179 & $75.0 \pm 4.0$ & I & Moresco et al. (2012)-[50] \\
\hline 0.199 & $75.0 \pm 5.0$ & I & Moresco et al. (2012)-[50] \\
\hline 0.2 & $72.9 \pm 29.6$ & I & Zhang et al. (2014)-[47] \\
\hline 0.240 & $79.69 \pm 2.65$ & II & Gaztañaga et al. (2009)-[51] \\
\hline 0.27 & $77.0 \pm 14.0$ & I & Simon et al. (2005)-[49] \\
\hline 0.28 & $88.8 \pm 36.6$ & I & Zhang et al. (2014)-[47] \\
\hline 0.35 & $84.4 \pm 7.0$ & II & Xu et al. (2013)-[52] \\
\hline 0.352 & $83.0 \pm 14.0$ & I & Moresco et al. (2012)-[50] \\
\hline 0.3802 & $83.0 \pm 13.5$ & I & Moresco et al. (2016)-[40] \\
\hline 0.4 & $95 \pm 17.0$ & I & Simon et al. (2005)-[49] \\
\hline 0.4004 & $77.0 \pm 10.2$ & I & Moresco et al. (2016)-[40] \\
\hline 0.4247 & $87.1 \pm 11.2$ & I & Moresco et al. (2016)-[40] \\
\hline 0.43 & $86.45 \pm 3.68$ & II & Gaztañaga et al. (2009)-[51] \\
\hline 0.44 & $82.6 \pm 7.8$ & II & Blake et al. (2012)-[53] \\
\hline 0.4497 & $92.8 \pm 12.9$ & $\mathrm{I}$ & Moresco et al. (2016)-[40] \\
\hline 0.47 & $89 \pm 50$ & I & Ratsimbazafy et al. (2017)-[54] \\
\hline 0.4783 & $80.9 \pm 9.0$ & I & Moresco et al. (2016)-[40] \\
\hline 0.48 & $97.0 \pm 62.0$ & I & Stern et al. (2010)-[55] \\
\hline 0.57 & $92.4 \pm 4.5$ & II & Samushia et al. (2013)-[56] \\
\hline 0.593 & $104.0 \pm 13.0$ & I & Moresco et al. (2012)-[50] \\
\hline 0.6 & $87.9 \pm 6.1$ & II & Blake et al. (2012)-[53] \\
\hline 0.68 & $92.0 \pm 8.0$ & I & Moresco et al. (2012)-[50] \\
\hline 0.73 & $97.3 \pm 7.0$ & II & Blake et al. (2012)-[53] \\
\hline 0.781 & $105.0 \pm 12.0$ & I & Moresco et al. (2012)-[50] \\
\hline 0.875 & $125.0 \pm 17.0$ & I & Moresco et al. (2012)-[50] \\
\hline 0.88 & $90.0 \pm 40.0$ & I & Stern et al. (2010)-[55] \\
\hline 0.9 & $117.0 \pm 23.0$ & I & Simon et al. (2005)-[49] \\
\hline 1.037 & $154.0 \pm 20.0$ & I & Moresco et al. (2012)-[50] \\
\hline 1.3 & $168.0 \pm 17.0$ & I & Simon et al. (2005)-[49] \\
\hline 1.363 & $160.0 \pm 33.6$ & I & Moresco (2015)-[57] \\
\hline 1.43 & $177.0 \pm 18.0$ & I & Simon et al. (2005)-[49] \\
\hline 1.53 & $140.0 \pm 14.0$ & I & Simon et al. (2005)-[49] \\
\hline 1.75 & $202.0 \pm 40.0$ & I & Simon et al. (2005)-[49] \\
\hline 1.965 & $186.5 \pm 50.4$ & I & Moresco (2015)-[57] \\
\hline 2.34 & $222.0 \pm 7.0$ & II & Delubac et al. (2015)-[58] \\
\hline 2.36 & $226.0 \pm 8.0$ & II & Font-Ribera et al. (2014)-[59] \\
\hline
\end{tabular}

$\overline{\mathrm{a}} \mathrm{km} \mathrm{s}^{-1} \mathrm{Mpc}^{-1}$. ${ }^{\mathrm{b}}$ Methods I and II represent the cosmic chronometers and the radial BAO size methods, respectively.

In consideration of the non-Gaussian posterior in parameter space, we perform a full Markov chain Monte Carlo (MCMC) Metropolis-Hastings sampling with emcee [60] and assume flat parameter priors to constrain these parameters, for $\mathrm{OHD}$ (or $H(z)$ data)

$$
\chi^{2}=\sum_{i} \frac{\left[H_{\mathrm{th}}\left(z_{i} \mid \boldsymbol{p}\right)-H_{\mathrm{obs}}\left(z_{i}\right)\right]^{2}}{\sigma^{2}\left(z_{i}\right)},
$$

in which $H_{\mathrm{th}}\left(z_{i} \mid \boldsymbol{p}\right), H_{\mathrm{obs}}\left(z_{i}\right)$, and $\sigma\left(z_{i}\right)$ are the theoretical Hubble parameter at redshift $z_{i}$, the OHD (or $H(z)$ data), and the uncertainty of each $H_{\mathrm{obs}}\left(z_{i}\right)$, respectively. For BAO, we use the same procedure given in Cao et al. [36]. We show in Tables 2 and 3 the unmarginalized and marginalized best-fitting results (the marigninalized ones are the posterior means) with 
$1 \sigma$ confidence regions, and with the best-fitting parameters (details described below), we can substitute Equation (15) into Equation (5) to obtain the age of the universe. Furthermore, panal (a) of Figure 1 shows theoretical curves of $H(z)$ for the best-fitting models along with the OHD. The $1 \sigma$ and $2 \sigma$ contours are shown in Figures $2-4$, which are analyzed by using the Python package GetDist [61]. Note that in Model 1, $\boldsymbol{p}=\left\{H_{0}, \Omega_{m_{0}}, w_{\mathrm{X}}, A_{1}, A_{2}\right\}$ for OHD and $\boldsymbol{p}=\left\{H_{0}, \Omega_{c_{0}} h^{2}, \Omega_{b_{0}} h^{2}, w_{\mathrm{X}}, A_{1}, A_{2}\right\}$ for $H(z)+$ BAO combination; in Models 2 and $3, \boldsymbol{p}=\left\{H_{0}, \Omega_{m_{0}}, w_{\mathrm{X}}, \lambda\right\}$ for OHD and $\boldsymbol{p}=\left\{H_{0}, \Omega_{c_{0}} h^{2}, \Omega_{b_{0}} h^{2}, w_{\mathrm{X}}, \lambda\right\}$ for $H(z)+$ BAO combination; the priors on $p$ are non-zero and flat over the ranges $0.005 \leq \Omega_{\mathrm{b}_{0}} h^{2} \leq 0.1$, $0.001 \leq \Omega_{\mathrm{C}_{0}} h^{2} \leq 0.99,0.2 \leq h \leq 1.0,-5 \leq w_{\mathrm{X}} \leq 0,0 \leq A_{1} \leq 2,0.2 \leq A_{2} \leq 10$, and $-5 \leq \lambda \leq 5$. We also include the Akaike Information Criterion (AIC) and the Bayesian Information Criterion $(B I C)$ to compare the goodness of fit of these models with different numbers of parameters, where

$$
A I C=\chi_{\min }^{2}+2 n,
$$

and

$$
B I C=\chi_{\min }^{2}+n \ln N,
$$

where $n$ and $N$ are the numbers of free parameters of the given model and of data points. For OHD, $N=40$, and for $H(z)+$ BAO combination, $N=42$. The degrees of freedom is $v=N-n$, which is used to determine the reduced $\chi^{2}$, i.e., $\chi_{\min }^{2} / v$ listed in Table 2 . We will discuss the details of these best-fitting models in detail in the following section.

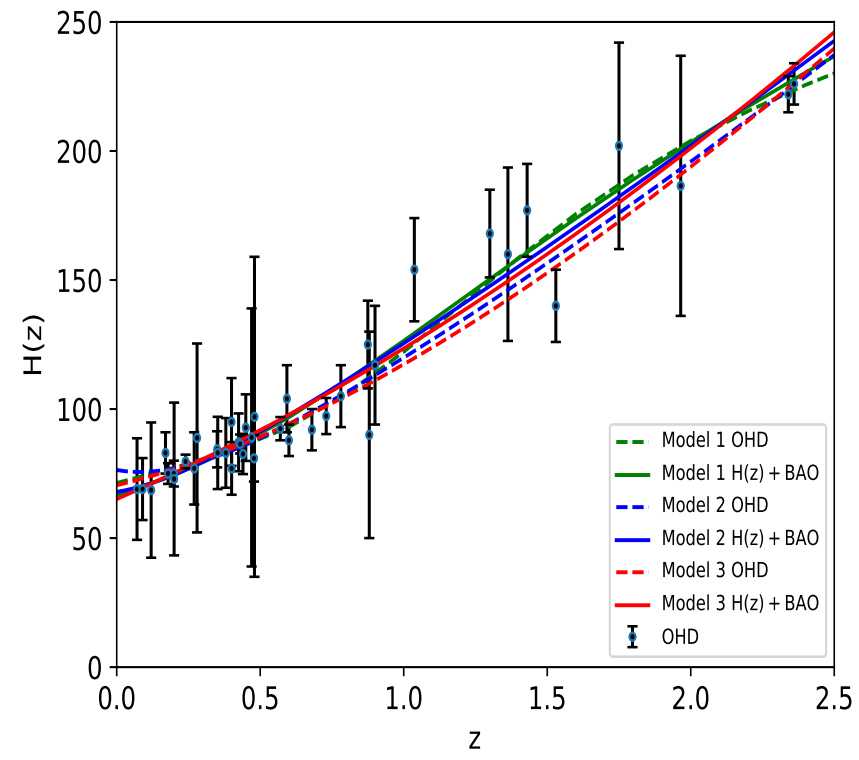

(a)

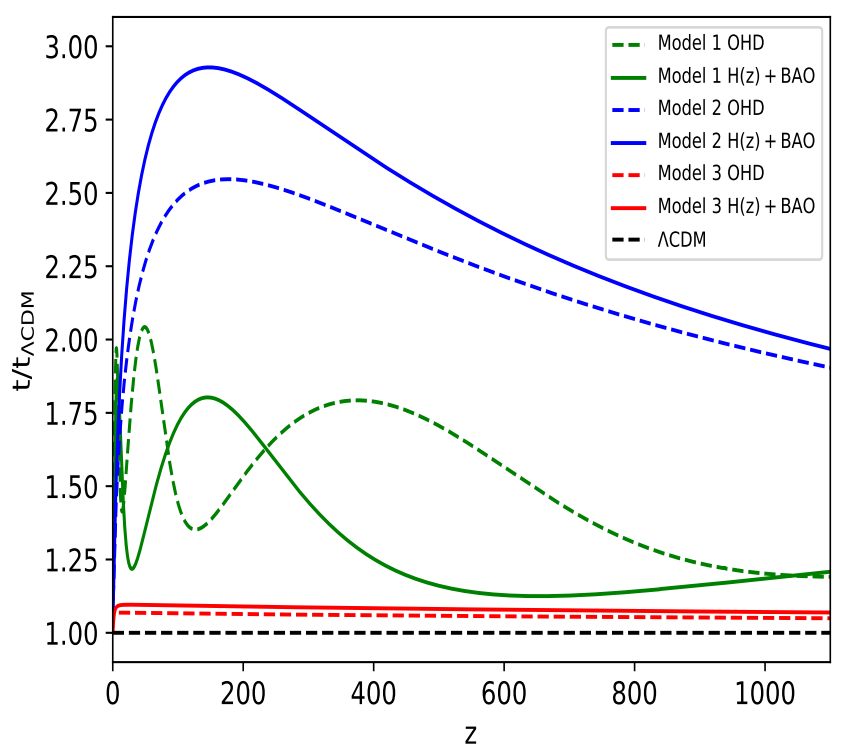

(b)

Figure 1. Hubble parameter $H(z)$ as a function of redshift $z$ for the coupling models with best-fitting parameters listed in Table 2, along with $\mathrm{OHD}(\mathbf{a})$, and the age ratio $t / t_{\Lambda \mathrm{CDM}}$ as a function of redshift $z(\mathbf{b})$, where the current values are 1.11 (1.08), 1.04 (1.04), and 1.02 (1.01) for Model 1, 2, and 3 constrained from OHD $(H(z)+$ BAO), respectively. 


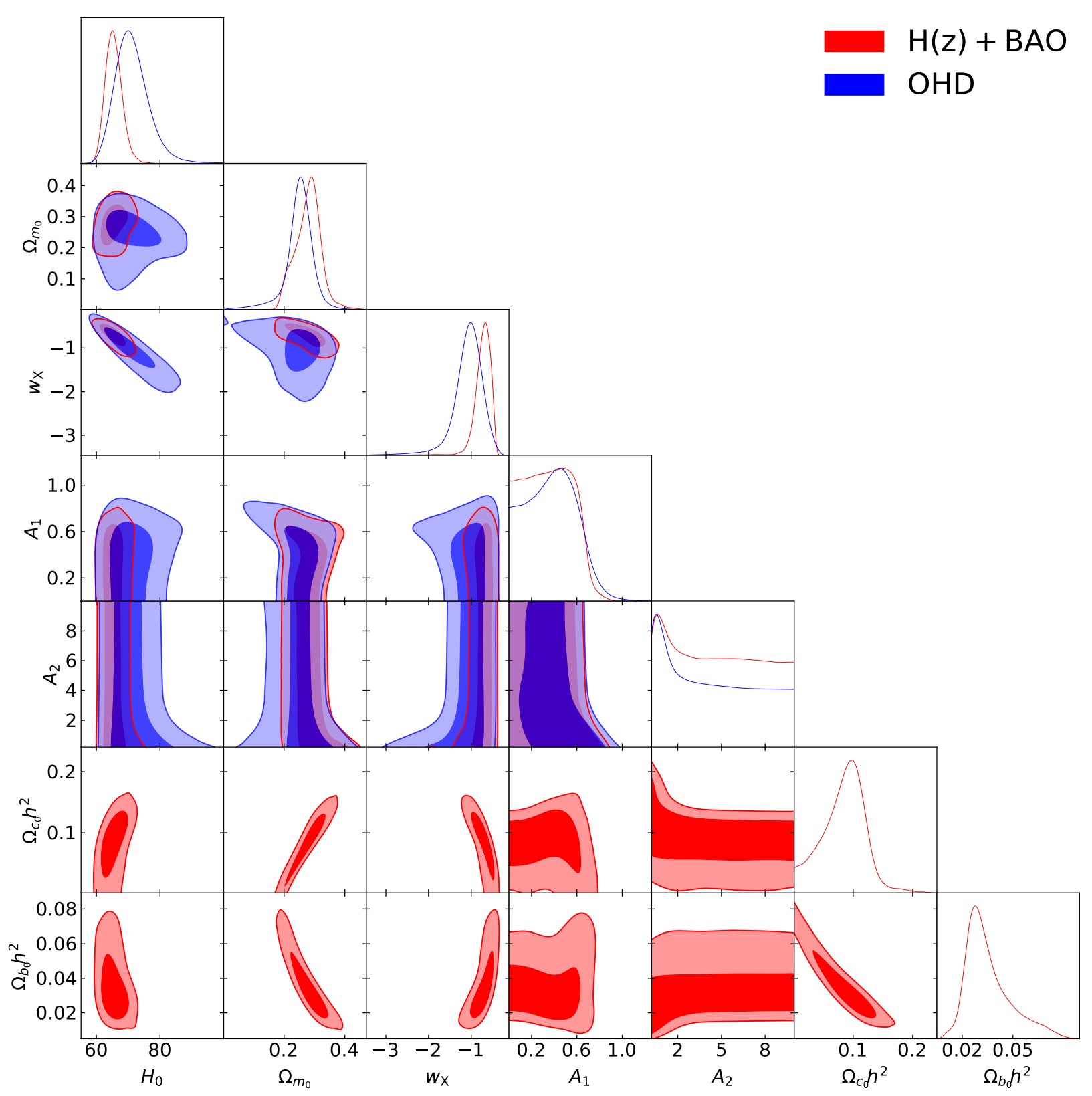

Figure 2. $1 \sigma$ and $2 \sigma$ contours for Model 1 with different data combinations with best-fitting results shown in Tables 2 and 3. 


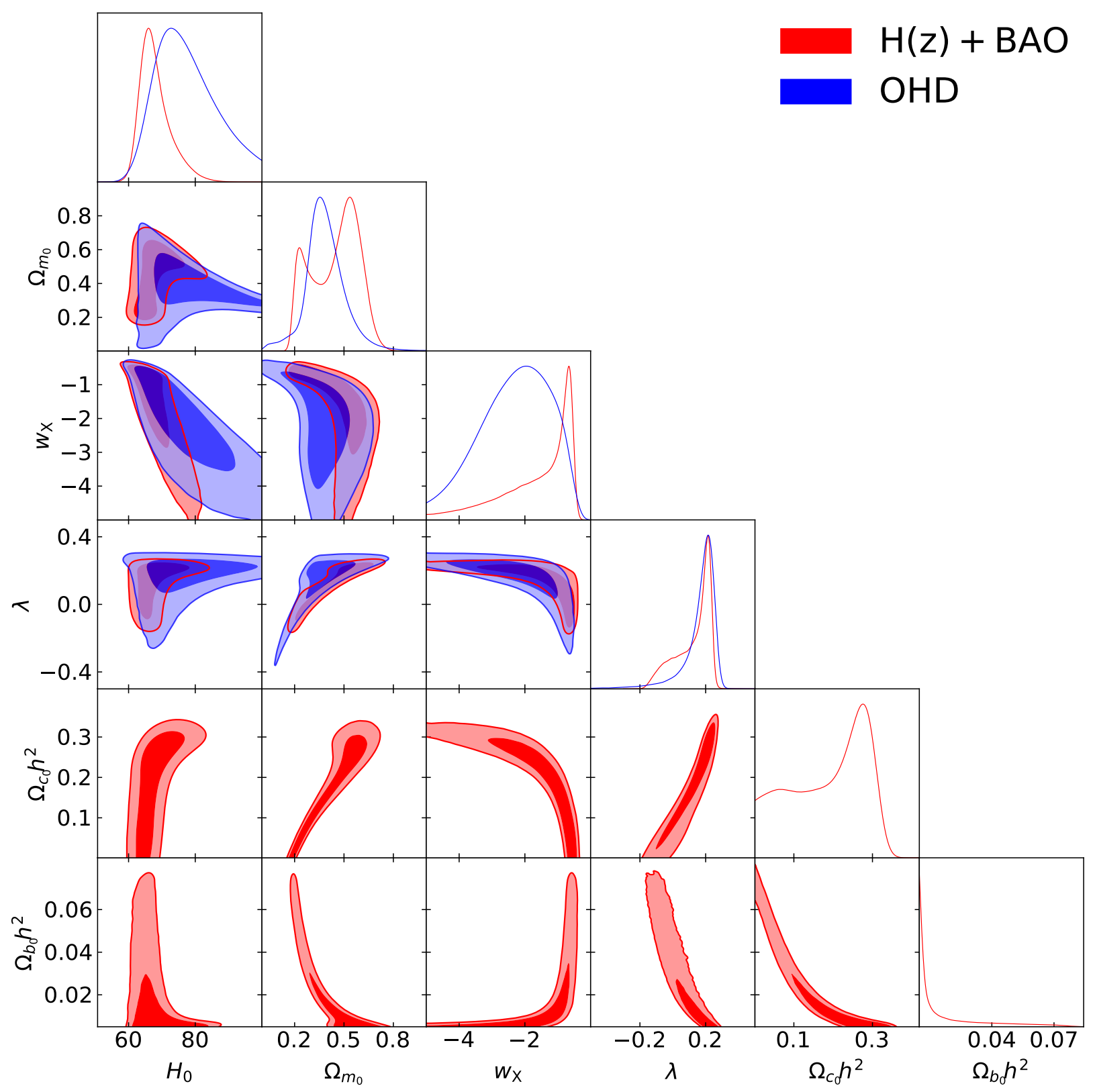

Figure 3. Same as Figure 2, but for Model 2. 


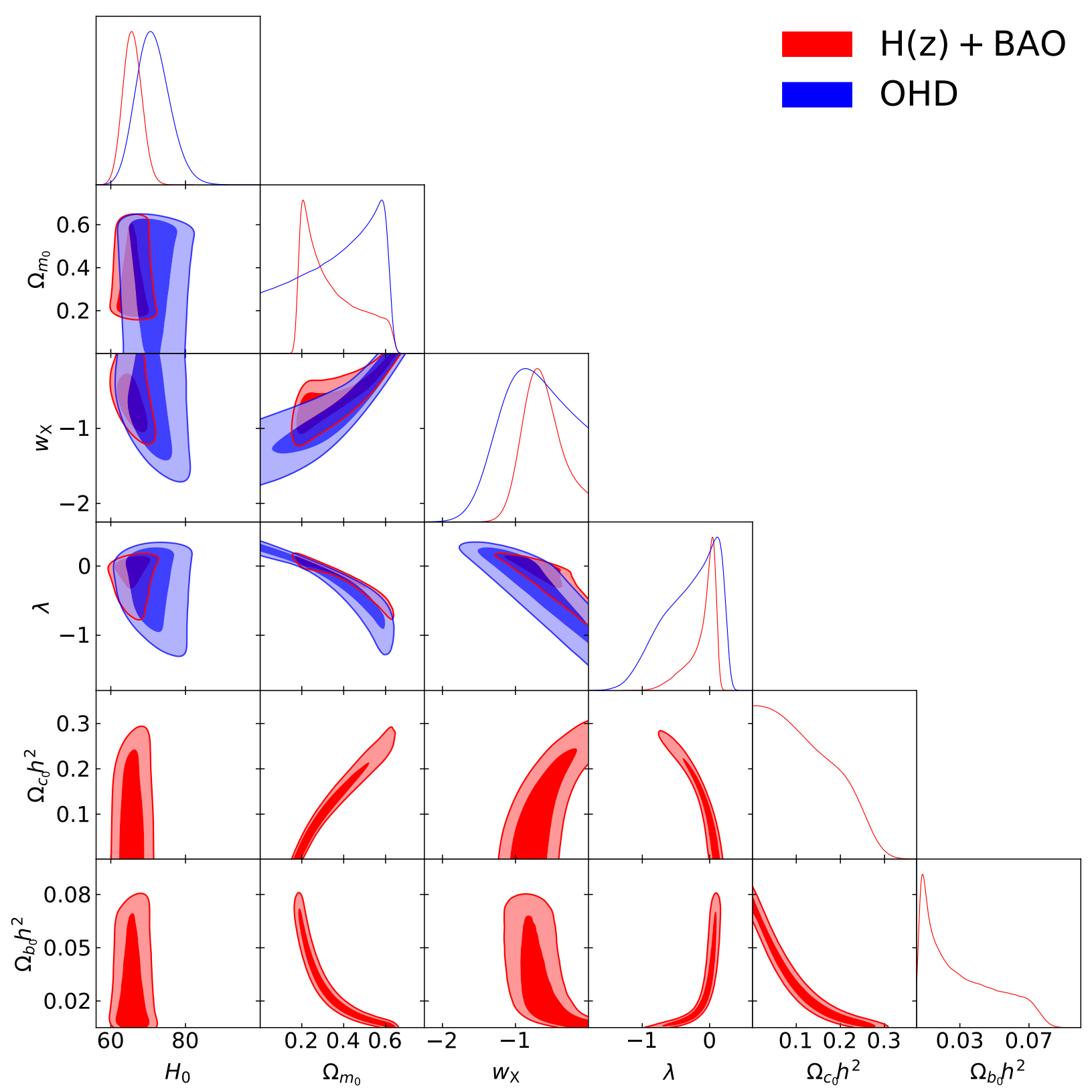

Figure 4. Same as Figure 2, but for Model 3.

\section{Preferred Best-Fitting Models and Their Implications}

From Table 2 we can see that although Model 1 has the lowest $\chi_{\min }^{2}$ based on AIC and $B I C$, Model 2 is the best candidate among these three models. The evidence against Model 1 is not strong, so Model 1 is not ruled out from these three candidate models. The values of reduced $\chi_{\min }^{2} \sim 0.5$ suggest that the error bars of these data are overestimated. Below we summarize the main results for the best-fitting models with the unmarginalized best-fitting parameters listed in Table 2 in different data combinations. Note that unmariginalized bestfitting parameters means that the parameters are given by maximizing the full likelihood, while the marginalized likelihood means the likelihood is obtained by marginalizing over one (or some) of the parameters.

Model 1 With the best-fitting parameters in this model, we have:

1. $\quad$ EoR $(6 \lesssim z \lesssim 15)$, for $\mathrm{OHD}(H(z)+\mathrm{BAO})$, from around $379.9 \mathrm{Myr}(402.6 \mathrm{Myr})$ to $1.825 \mathrm{Gyr}(1.718 \mathrm{Gyr})$, which lasts $~ 1.445 \mathrm{Gyr}(1.315 \mathrm{Gyr})$;

2. The seed redshift of J0100+2802, for OHD $\left(H(z)+\right.$ BAO), $z_{\text {seed }} \sim 9.74$ (11.03); 
3. If the first star formed at $z \approx 20$, then the age of the universe at that time would be, for OHD $(H(z)+$ BAO), 272.8 Myr (234.1 Myr);

4. Old globular cluster M92 (NGC 6341) would appear at $z \sim 7.53$ (9.27) with age of 14.0 Gyr for OHD $(H(z)+\mathrm{BAO})$;

5. For OHD $(H(z)+$ BAO), 3.5-Gyr-old ratio galaxy 53W091 $(z \sim 1.55)$ and 4Gyr-old radio galaxy 53W069 $(z \sim 1.43)$ are formed at $z \sim 4.98(5.40)$ and $z \sim 5.41$ (5.99), respectively;

6. For OHD $(H(z)+$ BAO), QSO APM 08279+5255 with age around 2.1 Gyr [62] would have formed at $z \sim 9.37$ (12.32).

Model 2 With the best-fitting parameters in this Model, we have:

1. $\quad \operatorname{EoR}(6 \lesssim z \lesssim 15)$, for $\mathrm{OHD}(H(z)+\mathrm{BAO})$, from around 478.7 Myr (534.1 Myr) to 1.371 Gyr (1.462 Gyr), which lasts 892.4 Myr (927.8 Myr);

2. The seed redshift of J0100+2802, for OHD $(H(z)+\mathrm{BAO}), z_{\text {seed }} \sim 17.75(16.46)$;

3. If the first star formed at $z \approx 20$, then the age of the universe at that time would be, for OHD $(H(z)+$ BAO), 337.1 Myr (381.4 Myr);

4. Old globular cluster M92 (NGC 6341) would appear at $z \sim 18.19$ (18.98) with age of 14.0 Gyr for OHD $(H(z)+\mathrm{BAO})$;

5. For OHD $(H(z)+$ BAO), 3.5-Gyr-old ratio galaxy 53W091 $(z \sim 1.55)$ and 4Gyr-old radio galaxy $53 W 069(z \sim 1.43)$ are formed at $z \sim 5.95(6.20)$ and $z \sim 6.85$ (7.22), respectively;

6. For OHD $(H(z)+$ BAO), QSO APM 08279+5255 with age around 2.1 Gyr would have formed at $z \sim 92.16$ (44.83). If we consider it to be formed after $z \approx 20$, then its age would be $\sim 1.810(1.861)$ Gyr for OHD $(H(z)+$ BAO).

Model 3 With the best-fitting parameters in this model, we have:

1. $\operatorname{EoR}(6 \lesssim z \lesssim 15)$, for $\mathrm{OHD}(H(z)+\mathrm{BAO})$, from around $286.8 \mathrm{Myr}(293.9 \mathrm{Myr})$ to $992.2 \mathrm{Myr}(1.011 \mathrm{Gyr})$, which lasts 705.4 Myr (716.8 Myr);

2. The seed redshift of J0100+2802, for OHD and $H(z)+\mathrm{BAO}, z_{\text {seed }}>20$;

3. If the first star formed at $z \approx 20$, then the age of the universe at that time would be, for OHD $(H(z)+$ BAO), 190.5 Myr (195.3 Myr);

4. Old globular cluster M92 (NGC 6341) would appear at $z \sim 71.70(>1100)$ with age of 14.0 Gyr for OHD and $H(z)+$ BAO;

5. addedFor $\operatorname{OHD}(H(z)+\mathrm{BAO}), 3.5-G y r-o l d$ ratio galaxy 53W091 $(z \sim 1.55)$ and 4-Gyr-old radio galaxy 53W069 $(z \sim 1.43)$ are formed at $z \sim 6.48(6.92)$ and $z \sim 7.76$ (8.54), respectively;

6. For OHD and $H(z)+$ BAO, QSO APM 08279+5255 with age around 2.1 Gyr would have formed at $z>1100$. (ruled out)

Table 2. Unmarginalized best-fitting parameter values for the coupling models.

\begin{tabular}{|c|c|c|c|c|c|c|c|c|c|c|c|c|c|c|c|c|}
\hline Model & Data Set & $\Omega_{c_{0}} h^{2}$ & $\Omega_{b_{0}} h^{2}$ & $\Omega_{m_{0}}$ & $w_{\mathrm{X}}$ & $H_{0}{ }^{\mathrm{a}}$ & $A_{1}$ & $A_{2}$ & $\lambda$ & $\chi_{\min }^{2}$ & $v^{\mathrm{b}}$ & $\chi_{\min }^{2} / v$ & $A I C$ & $B I C$ & $\Delta A I C$ & $\Delta B I C$ \\
\hline \multirow[t]{2}{*}{ Model 1} & OHD & - & - & 0.020 & -0.740 & 71.34 & 1.210 & 0.333 & - & 15.85 & 35 & 0.45 & 25.85 & 34.30 & 0.35 & 2.04 \\
\hline & $H(z)+\mathrm{BAO}$ & 0.0010 & 0.0742 & 0.171 & -0.797 & 66.64 & 0.907 & 0.472 & - & 17.85 & 36 & 0.50 & 29.85 & 40.27 & 1.43 & 3.16 \\
\hline \multirow[t]{2}{*}{ Model 2} & $\mathrm{OHD}$ & - & - & 0.383 & -1.904 & 76.39 & - & - & 0.167 & 17.50 & 36 & 0.49 & 25.50 & 32.26 & 0.00 & 0.00 \\
\hline & $H(z)+\mathrm{BA}$ & 0.259 & 0.006 & 0.579 & -1.973 & 67.90 & - & - & 0.206 & 18.42 & 37 & 0.50 & 28.42 & 37.11 & 0.00 & 0.00 \\
\hline \multirow[t]{2}{*}{ Model 3} & OHD & - & - & 0.157 & -1.107 & 70.48 & - & - & 0.112 & 18.47 & 36 & 0.51 & 26.47 & 33.23 & 0.97 & 0.97 \\
\hline & $H(z)+\mathrm{BA}$ & .133 & 0.019 & 0.361 & -0.607 & 65.12 & - & - & -0.088 & 19.65 & 37 & 0.53 & 29.65 & 38.34 & 1.23 & 1.23 \\
\hline
\end{tabular}


Table 3. One-dimensional marginalized best-fitting parameter values and uncertainties ( $\pm 1 \sigma$ or $2 \sigma$ upper limits) for the coupling models.

\begin{tabular}{|c|c|c|c|c|c|c|c|c|c|}
\hline Model & Data Set & $\Omega_{c_{0}} h^{2}$ & $\Omega_{b_{0}} h^{2}$ & $\Omega_{m_{0}}$ & $w_{\mathrm{X}}$ & $H_{0}{ }^{a}$ & $A_{1}$ & $A_{2}$ & $\lambda$ \\
\hline Model 1 & $\begin{array}{c}\text { OHD } \\
H(z)+\mathrm{BAO}\end{array}$ & $\begin{array}{c}- \\
0.0832_{-0.0300}^{+0.0402} \\
\end{array}$ & $\begin{array}{c}- \\
0.0361_{-0.0163}^{+0.0069}\end{array}$ & $\begin{array}{l}0.249_{-0.029}^{+0.042} \\
0.278_{-0.039}^{+0.045}\end{array}$ & $\begin{array}{r}-1.077_{-0.223}^{+0.347} \\
-0.734_{-0.095}^{+0.219} \\
\end{array}$ & $\begin{array}{r}71.28_{-5.85}^{+3.95} \\
65.50_{-2.86}^{+2.20} \\
\end{array}$ & $\begin{array}{r}0.376_{-0.243}^{+0.220} \\
0.359_{-0.215}^{+0.251} \\
\end{array}$ & $\begin{array}{l}4.521_{-4.295}^{+1.827} \\
4.771_{-4.510}^{+2.336} \\
\end{array}$ & - \\
\hline Model 2 & $\begin{array}{c}\mathrm{OHD} \\
H(z)+\mathrm{BAO}\end{array}$ & $\begin{array}{c}- \\
0.1899_{-0.1371}^{+0.1267} \\
\end{array}$ & $\begin{array}{c}- \\
<0.0628 \\
\end{array}$ & $\begin{array}{r}0.385_{-0.111}^{+0.093} \\
0.444_{-0.240}^{+0.183}\end{array}$ & $\begin{array}{r}-2.298_{-0.813}^{+1.355} \\
-1.650_{-0.396}^{+1.203} \\
\end{array}$ & $\begin{array}{c}77.42_{-11.21}^{+6.66} \\
68.04_{-5.45}^{+2.62} \\
\end{array}$ & - & - & $\begin{array}{r}0.158_{-0.019}^{+0.107} \\
0.127_{-0.049}^{+0.124} \\
\end{array}$ \\
\hline Model 3 & $\begin{array}{c}\text { OHD } \\
H(z)+\mathrm{BAO}\end{array}$ & $\begin{array}{c}- \\
<0.2532\end{array}$ & $\begin{array}{c}- \\
0.0314_{-0.0264}^{+0.0094}\end{array}$ & $\begin{array}{r}0.363_{-0.111}^{+0.255} \\
0.344_{-0.168}^{+0.065}\end{array}$ & $\begin{array}{r}0.754_{-0.439}^{+0.471} \\
-0.629_{-0.306}^{+0.220} \\
\end{array}$ & $\begin{array}{r}71.36_{-4.93}^{+3.93} \\
65.80_{-2.64}^{+2.38} \\
\end{array}$ & - & $\begin{array}{l}- \\
-\end{array}$ & $\begin{array}{r}0.293_{-0.230}^{+0.529} \\
-0.108_{-0.059}^{+0.235}\end{array}$ \\
\hline
\end{tabular}

Therefore, the Model $1 \mathrm{OHD}$ and $H(z)+\mathrm{BAO}$ cases are the best cases to alleviate the aging problems. In Figure 5, the redshifts when $H(z) / H_{\Lambda \mathrm{CDM}}(z) \sim 1$ are 0.40, 0.91, and 1.99 (Model 1 OHD), 0.30 and 1.96 (Model $1 \mathrm{H}(z)+$ BAO), 0.40 (Model 2 OHD), 0.04, 0.24, and 1.83 (Model $2 \mathrm{H}(z)+\mathrm{BAO}$ ), 0.52 and 725.79 (Model 3 OHD), and 0.16, 1.56, and 984.77 (Model $3 H(z)+\mathrm{BAO}$ ); and the current values of $H / H_{\Lambda \mathrm{CDM}}$ are 0.49 (Model 1 OHD), 0.33 (Model $1 H(z)+$ BAO), 0.49 (Model 2 OHD), 0.47 (Model $2 H(z)+$ BAO), 1.03 (Model $3 \mathrm{OHD}$ ), and 1.01 (Model $3 \mathrm{H}(z)+\mathrm{BAO}$ ). Note that the evolutions of dust matter density parameter $\Omega_{m}$ and DE density parameter $\Omega_{X}$ are shown in Figures 6 and 7 up to $z=1100$, where for Model $1 \mathrm{OHD}(H(z)+\mathrm{BAO})$, the redshifts when $\Omega_{m}$ have local extrema are 1.11, 4.26, 12.51, 41.89, 107.78, 356.20, and 928.41 (Model 1 OHD); 1.03, 4.72, 21.71, 115.98, and 455.68 (Model $1 \mathrm{H}(z)+\mathrm{BAO}$ ); 3.98 (Model 2 OHD); 3.23 (Model $1 \mathrm{H}(z)+$ BAO); 14.15 (Model 1 OHD); and 24.84 (Model $1 \mathrm{H}(z)+\mathrm{BAO}$ ); while the redshifts when $\Omega_{\mathrm{X}}$ have local extrema are 1.11, 4.24, 12.51, 40.53, 107.27, 300.45, and 906.09 (Model 1 OHD); 1.03, 4.70, 21.67, 108.17, and 443.89 (Model $1 H(z)+$ BAO); and none for the rest cases. Since the universe has a slightly larger age in these best-fitting models, we also care about how it works with the expansion of the universe. The deceleration parameter is

$$
q(z)=-\left(\frac{\ddot{a}}{a}\right) / H^{2}=\frac{1}{H(z)} \frac{d H(z)}{d z}(1+z)-1 .
$$

As shown in Figure 8, except for the Model 3 OHD and $H(z)+$ BAO cases, the deceleration parameter $q(z)$ for the other four cases are going through transitions from deceleration to acceleration phases. For the Model 1 OHD case, the transition redshifts (for $z \leq 1100$ ) are 8.67, 56.70, and 169.15; for the Model $1 \mathrm{H}(z)+\mathrm{BAO}$ case, the transition redshifts are 8.67, 56.70, and 169.15; for the Model 2 OHD case, the transition redshift is 0.45; and for the Model $2 \mathrm{H}(z)+\mathrm{BAO}$ case, the transition redshift is 0.64; while for the Model $3 \mathrm{OHD}$ and $H(z)+$ BAO cases, $q(z)<0$ is satisfied until today.

Although the Model 1 OHD and $H(z)+$ BAO cases are capable of explaining the aging issues, Model 1 have some strange (non-physical) behaviors due to the unbounded time-dependent choice of $\lambda$, where the matter density parameter $\Omega_{m}$ and the DE density parameter $\Omega_{X}$ oscillate with the value of $\Omega_{m}$ and $\Omega_{X}$ being a high of 2.51 (1.74) and a low of $-1.52(-0.75)$ for the Model 1 OHD and $H(z)+$ BAO cases, respectively. From panel (b) of Figure 1 we see that the Model 2 cases have relatively larger ages during the evolution and find that other than the age of QSO APM $08279+5255$, the other age issues are significantly improved. Furthermore, judging from AIC and BIC listed in Table 2, Model 2 is the most favored model among these three models. Therefore, we conclude that Model 2 is the best model. 


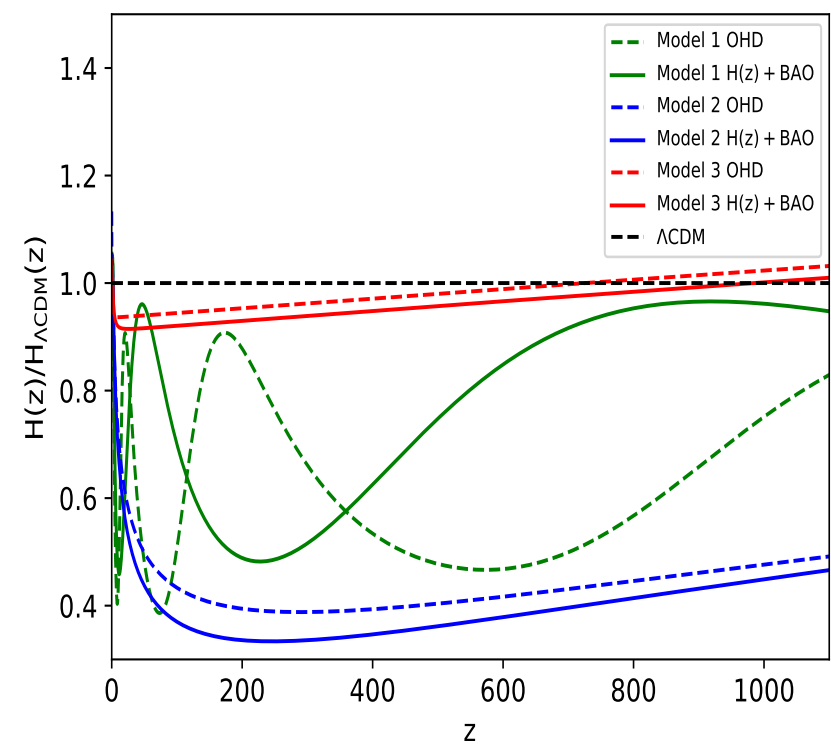

(a)

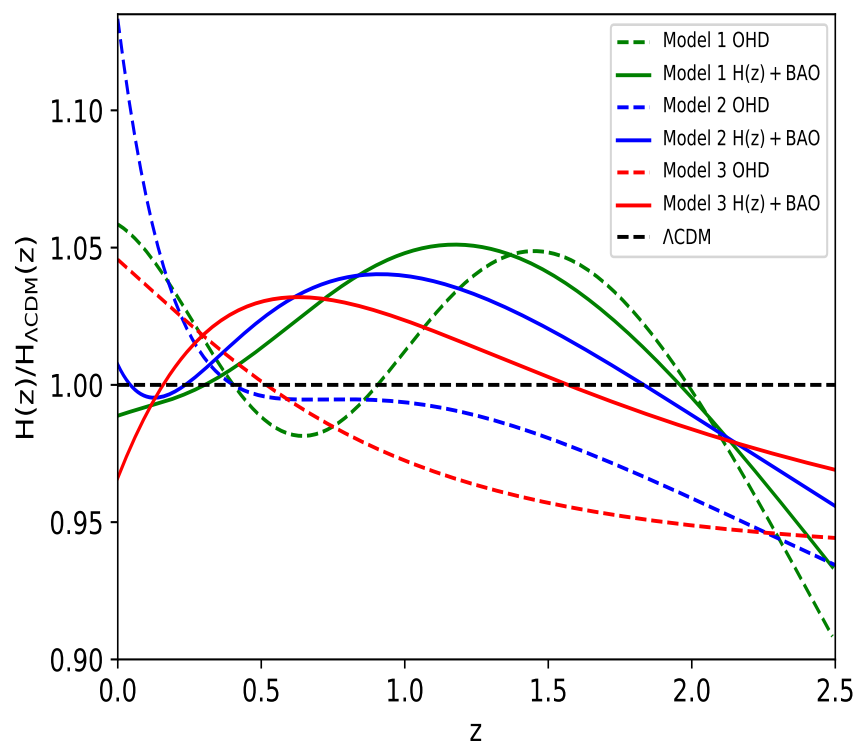

(b)

Figure 5. The ratio between Hubble parameter $H(z)$ of coupling models and that of $\Lambda$ CDM against redshift $z(\mathbf{a})$ and the (b) shows that of redshift range $0 \leq z \leq 2.5$.

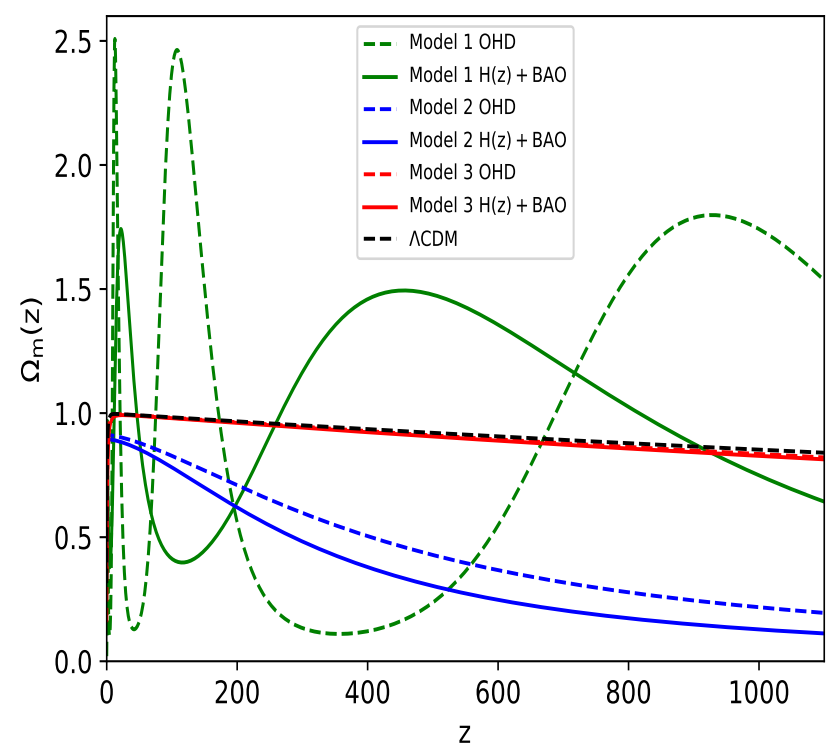

(a)

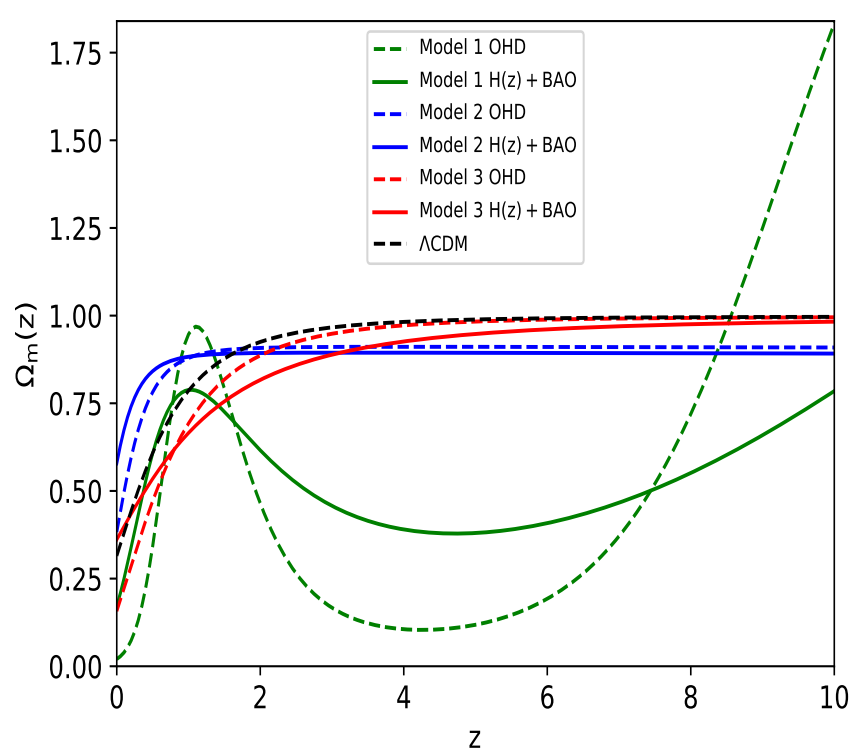

(b)

Figure 6. The cosmological matter density parameter $\Omega_{m}$ as a function of redshift $z$ (a) and the (b) shows that of redshift range $0 \leq z \leq 10$. 


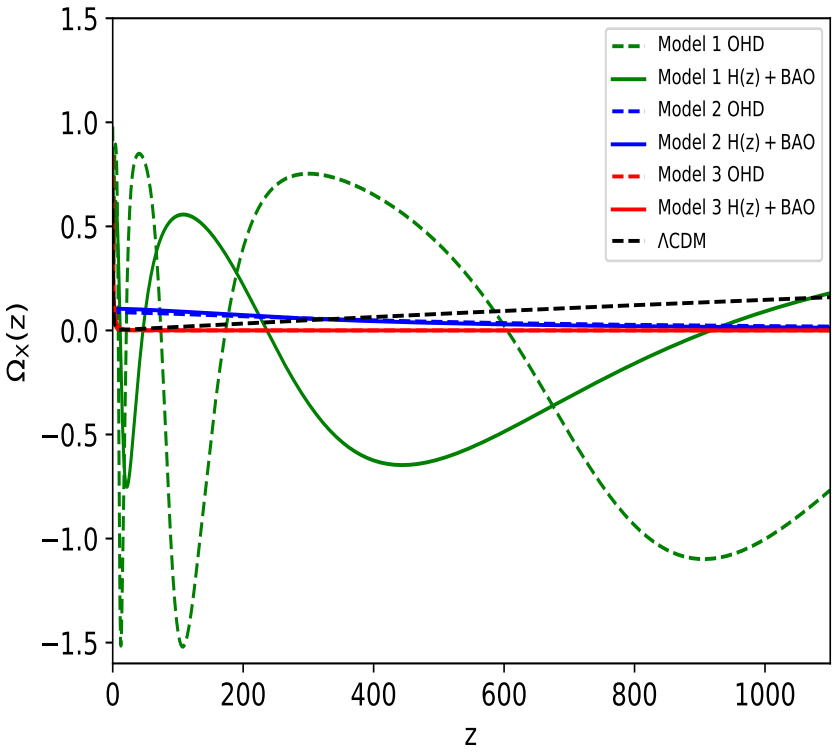

(a)

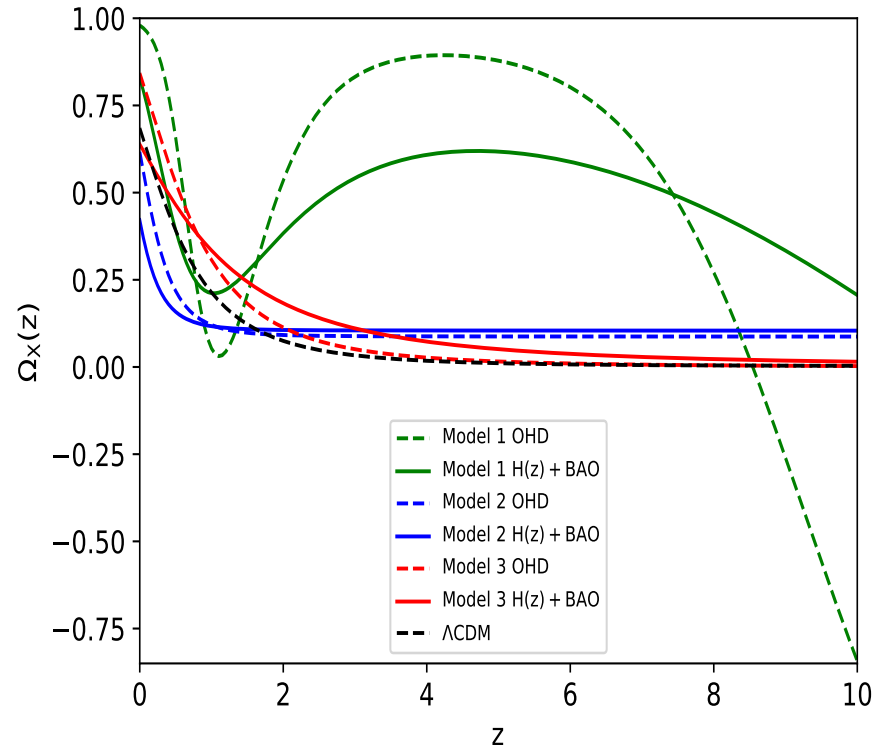

(b)

Figure 7. The cosmological dark energy density parameter $\Omega_{X}$ as a function of redshift $z$ (a) and the (b) shows that of redshift range $0 \leq z \leq 10$.

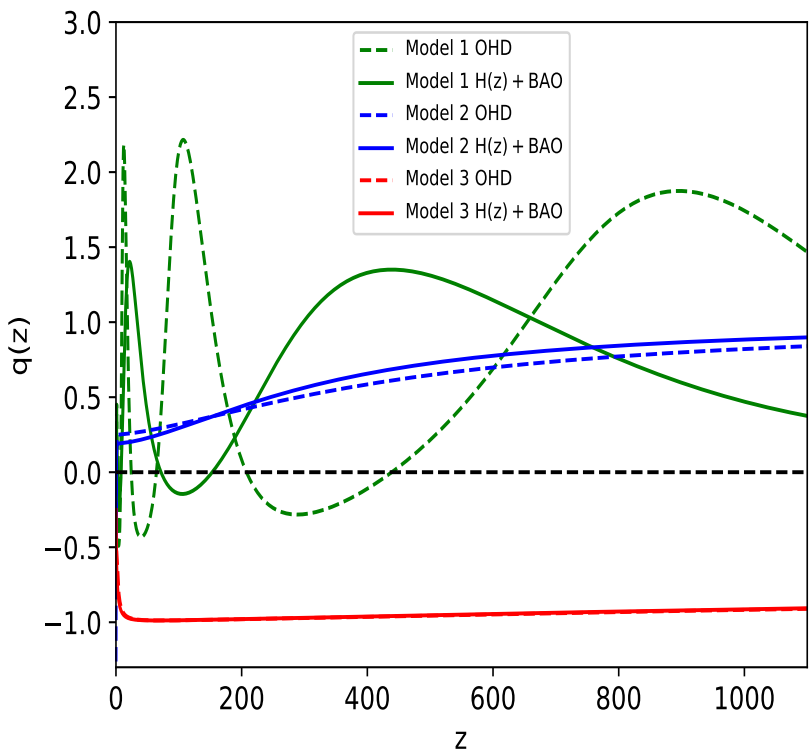

(a)

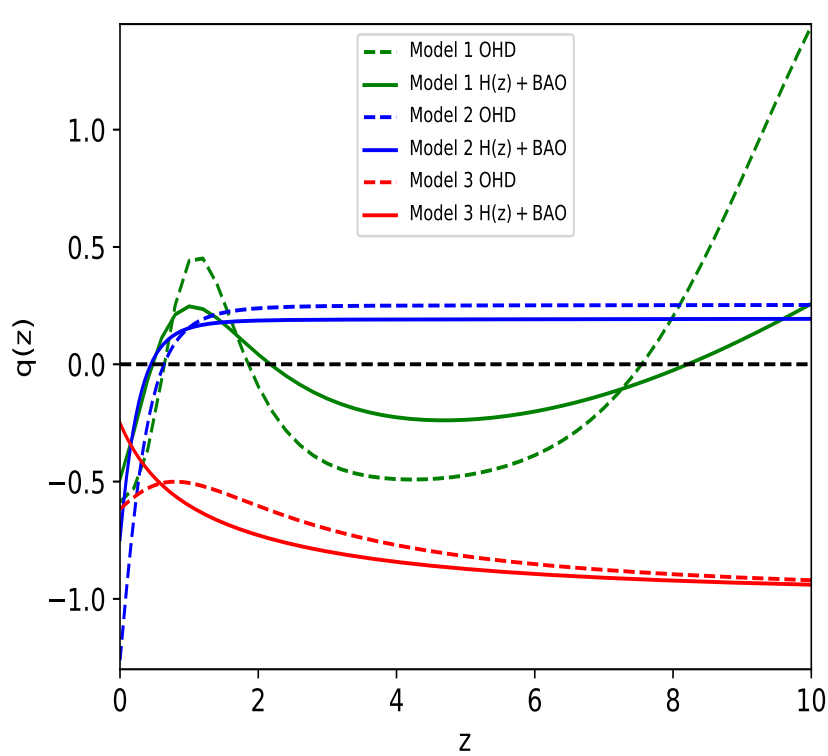

(b)

Figure 8. The deceleration parameter $q(z)$ as a function of redshift $z(\mathbf{a})$ and the (b) shows that of redshift range $0 \leq z \leq 10$.

\section{Conclusions and Discussions}

By exploring the properties of the coupling models with the best-fitting parameters obtained from constraints over $\mathrm{OHD}$ and $\mathrm{H}(z)+\mathrm{BAO}$, we manage to improve the aging problems of the $\Lambda \mathrm{CDM}$ model, where four of the six best-fitting models demonstrate good improvements on them. Although the Model 1 OHD and $H(z)+$ BAO cases have better capability of improving the issues, Model 1 has some strange (non-physical) behaviors (see, e.g., Figures 6 and 7). The Model $2 \mathrm{OHD}$ and $H(z)+$ BAO cases can alleviate most of the aging problems and based on AIC and BIC listed in Table 2, Model 2 is considered to be the best candidate model amongst these three models. For Model 2 $\mathrm{OHD}(H(z)+\mathrm{BAO})$, the constraints on the parameters are $\Omega_{m_{0}}=0.385_{-0.111}^{+0.093}\left(0.444_{-0.240}^{+0.183}\right)$, 
$w_{\mathrm{X}}=-2.298_{-0.813}^{+1.355}\left(-1.650_{-0.396}^{+1.203}\right), H_{0}=77.42_{-11.21}^{+6.66}\left(68.04_{-5.45}^{+2.62}\right) \mathrm{km} \mathrm{s}^{-1} \mathrm{Mpc}^{-1}$, and $\lambda=$ $0.158_{-0.019}^{+0.107}\left(0.127_{-0.049}^{+0.124}\right)$. The Model 2 OHD and $H(z)+$ BAO cases support the currently accelerating expansion of the the Universe with the expansions transitioned from decelerating to accelerating at redshits 0.45 and 0.64 , respectively. The ages of the universes are 14.385 Gyr and 14.409 Gyr in the Model 2 OHD and $H(z)+$ BAO cases, respectively. That Model 3 is ruled out does not convey the idea that the coupling between DE and DM is not worth investigating, because we do not include perturbations in this paper.

Author Contributions: Conceptualization, S.C. and T.-J.Z.; writing-original draft preparation, S.C. and X.W.; analysis and plotting, S.C. and T.Z.; writing-review and editing, T.-J.Z., X.W. and T.Z. All authors have read and agreed to the published version of the manuscript.

Funding: This work was funded by the National Science Foundation of China (Grants No.11929301, 61802428, 11573006) and National Key R\&D Program of China (2017YFA0402600).

Data Availability Statement: Not applicable.

Acknowledgments: We thank the anonymous referees for insightful suggestions that improved the paper significantly, and Qiang $\mathrm{Xu}$ and Zhongxu Zhai for useful ideas and discussions.

Conflicts of Interest: The authors declare no conflict of interest.

\section{References}

1. Mortonson, M.J.; Weinberg, D.H.; White, M. Dark Energy: A Short Review. arXiv 2013, arXiv:1401.0046.

2. Brax, P. What makes the Universe accelerate? A review on what dark energy could be and how to test it. Rep. Prog. Phys. 2017, 81, 016902. [CrossRef] [PubMed]

3. Arun, K.; Gudennavar, S.; Sivaram, C. Dark matter, dark energy, and alternate models: A review. Adv. Space Res. 2017, 60, 166-186. [CrossRef]

4. Peebles, P.J.E. Tests of cosmological models constrained by inflation. Astrophys. J. 1984, 284, 439-444. [CrossRef]

5. Sahni, V.; Shtanov, Y. Did the universe loiter at high redshifts? Phys. Rev. D 2005, 71, 084018. [CrossRef]

6. Richards, G.T.; Strauss, M.A.; Pindor, B.; Haiman, Z.; Fan, X.; Eisenstein, D.; Schneider, D.P.; Bahcall, N.A.; Brinkmann, J.; Brunner, R.; et al. A Snapshot Survey for Gravitational Lenses among $z \geq 4.0$ Quasars. I. The $z>5.7$ Sample. Astron. J. 2004, 127, 1305-1312. [CrossRef]

7. Haiman, Z.; Quataert, E. The Formation and Evolution of the First Massive Black Holes. In Supermassive Black Holes in the Distant Universe; Astrophysics and Space Science Library; Barger, A.J., Ed.; Springer: Dordrecht, The Netherlands, 2004; Volume 308, p. 147,

8. Melia, F.; McClintock, T.M. Supermassive black holes in the early Universe. Proc. R. Soc. Lond. Ser. A 2015, 471, 20150449,

9. Wu, X.B.; Wang, F.; Fan, X. An ultraluminous quasar with a twelve-billion-solar-mass black hole at redshift 6.30. Nature 2015, 518, 512-515. [CrossRef]

10. Pont, F.; Mayor, M.; Turon, C.; Vandenberg, D.A. HIPPARCOS subdwarfs and globular cluster ages: The distance and age of M 92. Astron. Astrophys. 1998, 329, 87-100.

11. Aghanim, N.; Akrami, Y.; Ashdown, M.; Aumont, J.; Baccigalupi, C.; Ballardini, M.; Banday, A.J.; Barreiro, R.B.; Bartolo, N.; Basak, S.; et al. Planck 2018 results. VI. Cosmological parameters. arXiv 2018, arXiv:1807.06209.

12. Dunlop, J.; Peacock, J.; Spinrad, H.; Dey, A.; Jimenez, R.; Stern, D.; Windhorst, R. A 3.5-Gyr-old galaxy at redshift 1.55. Nature 1996, 381, 581-584. [CrossRef]

13. Spinrad, H.; Dey, A.; Stern, D.; Dunlop, J.; Peacock, J.; Jimenez, R.; Windhorst, R. LBDS 53W091: An Old, Red Galaxy at z = 1.552. Astrophys. J. 1997, 484, 581-601. [CrossRef]

14. Wei, H.; Zhang, S.N. Interacting energy components and observational H(z) data. Phys. Lett. B 2007, 654, 139-147. [CrossRef]

15. Wei, H.; Zhang, S.N. Observational data and cosmological models. Phys. Lett. B 2007, 644, 7-15. [CrossRef]

16. Wei, H.; Cai, R.G. Interacting vectorlike dark energy, the first and second cosmological coincidence problems. Phys. Rev. D 2006, 73, 083002. [CrossRef]

17. Gannouji, R.; Moraes, B.; Mota, D.F.; Polarski, D.; Tsujikawa, S.; Winther, H.A. Chameleon dark energy models with characteristic signatures. Phys. Rev. D 2010, 82, 124006. [CrossRef]

18. Avilés, A.; Cervantes-Cota, J.L. Dark matter from dark energy-baryonic matter couplings. Phys. Rev. D 2011, 83, 023510. [CrossRef]

19. Khoury, J.; Weltman, A. Chameleon cosmology. Phys. Rev. D 2004, 69, 044026. [CrossRef]

20. Kumar, S.; Nunes, R.C. Echo of interactions in the dark sector. Phys. Rev. D 2017, 96, 103511. [CrossRef]

21. Di Valentino, E.; Melchiorri, A.; Mena, O. Can interacting dark energy solve the $\mathrm{H}_{0}$ tension? Phys. Rev. D 2017, 96, 043503. [CrossRef] 
22. An, R.; Feng, C.; Wang, B. Relieving the tension between weak lensing and cosmic microwave background with interacting dark matter and dark energy models. J. Cosmol. Astropart. Phys. 2018, 2018, 038. [CrossRef]

23. Yang, W.; Mukherjee, A.; Di Valentino, E.; Pan, S. Interacting dark energy with time varying equation of state and the $\mathrm{H}_{0}$ tension. Phys. Rev. D 2018, 98, 123527. [CrossRef]

24. Kumar, S.; Nunes, R.C.; Yadav, S.K. Dark sector interaction: A remedy of the tensions between CMB and LSS data. Eur. Phys. J. C 2019, 79, 576. [CrossRef]

25. Pan, S.; Yang, W.; Singha, C.; Saridakis, E.N. Observational constraints on sign-changeable interaction models and alleviation of the $\mathrm{H}_{0}$ tension. Phys. Rev. D 2019, 100, 083539. [CrossRef]

26. Di Valentino, E.; Melchiorri, A.; Mena, O.; Vagnozzi, S. Interacting dark energy in the early 2020s: A promising solution to the $\mathrm{H}_{0}$ and cosmic shear tensions. Phys. Dark Universe 2020, 30, 100666. [CrossRef]

27. Benoit, A.; Ade, P.; Amblard, A.; Ansari, R.; Aubourg, E.; Bargot, S.; Bartlett, J.G.; Bhatia, R.S.; Blanchard, A.; Bock, J.J.; et al. Cosmological constraints from Archeops. Astro. Astrophys. 2003, 399, L25-L30. [CrossRef]

28. Particle Data Group. Review of Particle Physics. Prog. Theor. Exp. Phys. 2020, 2020, 083C01. [CrossRef]

29. Zaroubi, S. The Epoch of Reionization. In The First Galaxies; Astrophysics and Space Science Library; Wiklind, T., Mobasher, B., Bromm, V., Eds.; Springer: Berlin/Heidelberg, Germany, 2013; Volume 396, p. 45,

30. Bromm, V.; Yoshida, N.; Hernquist, L.; McKee, C.F. The formation of the first stars and galaxies. Nature 2009, 459, 49-54. [CrossRef] [PubMed]

31. Bromm, V.; Coppi, P.S.; Larson, R.B. The Formation of the First Stars. I. The Primordial Star-forming Cloud. Astrophys. J. 2002, 564, 23-51. [CrossRef]

32. Nunes, R.C.; Pacucci, F. Effects of the Hubble parameter on the cosmic growth of the first quasars. Mon. Not. R. Astron. Soc. 2020, 496, 888-893. [CrossRef]

33. Bolotin, Y.L.; Kostenko, A.; Lemets, O.A.; Yerokhin, D.A. Cosmological evolution with interaction between dark energy and dark matter. Int. J. Mod. Phys. D 2015, 24, 1530007,

34. Ryan, J.; Doshi, S.; Ratra, B. Constraints on dark energy dynamics and spatial curvature from Hubble parameter and baryon acoustic oscillation data. Mon. Not. R. Astron. Soc. 2018, 480, 759-767. [CrossRef]

35. Ryan, J.; Chen, Y.; Ratra, B. Baryon acoustic oscillation, Hubble parameter, and angular size measurement constraints on the Hubble constant, dark energy dynamics, and spatial curvature. Mon. Not. R. Astron. Soc. 2019, 488, 3844-3856. [CrossRef]

36. Cao, S.; Ryan, J.; Ratra, B. Cosmological constraints from HII starburst galaxy apparent magnitude and other cosmological measurements. Mon. Not. R. Astron. Soc. 2020, 497, 3191-3203. [CrossRef]

37. Cao, S.; Ryan, J.; Khadka, N.; Ratra, B. Cosmological constraints from higher-redshift gamma-ray burst, H II starburst galaxy, and quasar (and other) data. Mon. Not. R. Astron. Soc. 2021, 501, 1520-1538. [CrossRef]

38. Cao, S.; Ryan, J.; Ratra, B. Using Pantheon and DES supernova, baryon acoustic oscillation, and Hubble parameter data to constrain the Hubble constant, dark energy dynamics, and spatial curvature. arXiv 2021, arXiv:2101.08817.

39. Moresco, M.; Cimatti, A.; Jimenez, R.; Pozzetti, L.; Zamorani, G.; Bolzonella, M.; Dunlop, J.; Lamareille, F.; Mignoli, M.; Pearce, $\mathrm{H}$; ; et al. Improved constraints on the expansion rate of the Universe up to $\mathrm{z}^{\sim} 1.1$ from the spectroscopic evolution of cosmic chronometers. J. Cosmol. Astropart. Phys. 2012, 8, 006. [CrossRef]

40. Moresco, M.; Pozzetti, L.; Cimatti, A.; Jimenez, R.; Maraston, C.; Verde, L.; Thomas, D.; Citro, A.; Tojeiro, R.; Wilkinson, D. A 6\% measurement of the Hubble parameter at $\mathrm{z}^{\sim} 0.45$ : direct evidence of the epoch of cosmic re-acceleration. J. Cosmol. Astropart. Phys. 2016, 5, 014. [CrossRef]

41. Moresco, M.; Jimenez, R.; Verde, L.; Pozzetti, L.; Cimatti, A.; Citro, A. Setting the Stage for Cosmic Chronometers. I. Assessing the Impact of Young Stellar Populations on Hubble Parameter Measurements. Astrophys. J. 2018, 868, 84. [CrossRef]

42. Moresco, M.; Jimenez, R.; Verde, L.; Cimatti, A.; Pozzetti, L. Setting the Stage for Cosmic Chronometers. II. Impact of Stellar Population Synthesis Models Systematics and Full Covariance Matrix. Astrophys. J. 2020, 898, 82. [CrossRef]

43. Alam, S.; Ata, M.; Bailey, S.; Beutler, F.; Bizyaev, D.; Blazek, J.A.; Bolton, A.S.; Brownstein, J.R.; Burden, A.; Chuang, C.H.; et al. The clustering of galaxies in the completed SDSS-III Baryon Oscillation Spectroscopic Survey: Cosmological analysis of the DR12 galaxy sample. Mon. Not. R. Astron. Soc. 2017, 470, 2617-2652. [CrossRef]

44. Cao, S.L.; Duan, X.W.; Meng, X.L.; Zhang, T.J. Cosmological model-independent test of $\Lambda$ CDM with two-point diagnostic by the observational Hubble parameter data. Eur. Phys. J. C 2018, 78, 313. [CrossRef]

45. Cao, S.L.; Teng, H.Y.; Wan, H.Y.; Yu, H.R.; Zhang, T.J. Testing backreaction effects with observational Hubble parameter data. Eur. Phys. J. C 2018, 78, 170. [CrossRef]

46. Cao, S.L.; Li, S.; Yu, H.R.; Zhang, T.J. Statefinder diagnostic and constraints on the Palatini f(R) gravity theories. Res. Astron. Astrophys. 2018, 18, 026,

47. Zhang, C.; Zhang, H.; Yuan, S.; Liu, S.; Zhang, T.J.; Sun, Y.C. Four new observational H(z) data from luminous red galaxies in the Sloan Digital Sky Survey data release seven. Res. Astron. Astrophys. 2014, 14, 1221-1233. [CrossRef]

48. Jimenez, R.; Verde, L.; Treu, T.; Stern, D. Constraints on the equation of state of dark energy and the Hubble constant from stellar ages and the CMB. Astrophys. J. 2003, 593, 622-629. [CrossRef]

49. Simon, J.; Verde, L.; Jimenez, R. Constraints on the redshift dependence of the dark energy potential. Phys. Rev. D Part. Fields, Gravit. Cosmol. 2005, 71, 123001, 
50. Moresco, M.; Verde, L.; Pozzetti, L.; Jimenez, R.; Cimatti, A. New constraints on cosmological parameters and neutrino properties using the expansion rate of the Universe to $\mathrm{z} \sim 1.75$. J. Cosmol. Astropart. Phys. 2012, 2012, 053. [CrossRef]

51. Gaztañaga, E.; Cabré, A.; Hui, L. Clustering of luminous red galaxies - IV. Baryon acoustic peak in the line-of-sight direction and a direct measurement of $\mathrm{H}(\mathrm{z})$. Mon. Not. R. Astron. Soc. 2009, 399, 1663-1680. [CrossRef]

52. Xu, X.; Cuesta, A.J.; Padmanabhan, N.; Eisenstein, D.J.; McBride, C.K. Measuring $\mathrm{D}_{A}$ and $\mathrm{H}$ at $\mathrm{z}=0.35$ from the SDSS DR7 LRGs using baryon acoustic oscillations. Mon. Not. R. Astron. Soc. 2013, 431, 2834-2860. [CrossRef]

53. Blake, C.; Brough, S.; Colless, M.; Contreras, C.; Couch, W.; Croom, S.; Croton, D.; Davis, T.M.; Drinkwater, M.J.; Forster, K.; et al. The WiggleZ Dark Energy Survey: Joint measurements of the expansion and growth history at $z<1$. Mon. Not. R. Astron. Soc. 2012, 425, 405-414.

54. Ratsimbazafy, A.L.; Loubser, S.I.; Crawford, S.M.; Cress, C.M.; Bassett, B.A.; Nichol, R.C.; Väisänen, P. Age-dating luminous red galaxies observed with the Southern African Large Telescope. Mon. Not. R. Astron. Soc. 2017, 467, 3239-3254. [CrossRef]

55. Stern, D.; Jimenez, R.; Verde, L.; Kamionkowski, M.; Stanford, S.A. Cosmic chronometers: Constraining the equation of state of dark energy. I: H(z) measurements. J. Cosmol. Astropart. Phys. 2010, 2010, 8. [CrossRef]

56. Samushia, L.; Reid, B.A.; White, M.; Percival, W.J.; Cuesta, A.J.; Lombriser, L.; Manera, M.; Nichol, R.C.; Schneider, D.P.; Bizyaev, D.; et al. The clustering of galaxies in the SDSS-III DR9 baryon oscillation spectroscopic survey: Testing deviations from $\Lambda$ and general relativity using anisotropic clustering of galaxies. Mon. Not. R. Astron. Soc. 2013, 429, 1514-1528. [CrossRef]

57. Moresco, M. Raising the bar: New constraints on the Hubble parameter with cosmic chronometers at z $\sim 2$. Mon. Not. R. Astron. Soc. Lett. 2015, 450, L16-L20,

58. Delubac, T.; Bautista, J.E.; Rich, J.; Kirkby, D.; Bailey, S.; Font-Ribera, A.; Slosar, A.; Lee, K.G.; Pieri, M.M.; Hamilton, J.C.; et al. Baryon Acoustic Oscillations in the Ly $\alpha$ forest of BOSS DR11 quasars. Astron. Astrophys. 2015, 574, A59. [CrossRef]

59. Font-Ribera, A.; Kirkby, D.; Miralda-Escudé, J.; Ross, N.P.; Slosar, A.; Rich, J.; Aubourg, É; Bailey, S.; Bhardwaj, V.; Bautista, J.; et al. Quasar-Lyman $\alpha$ forest cross-correlation from BOSS DR11: Baryon Acoustic Oscillations. J. Cosmol. Astropart. Phys. 2014, 2014, 027. [CrossRef]

60. Foreman-Mackey, D.; Hogg, D.W.; Lang, D.; Goodman, J. emcee: The MCMC Hammer. Publ. Astron. Soc. Pac. 2013, 125, 306. [CrossRef]

61. Lewis, A. GetDist: A Python package for analysing Monte Carlo samples. arXiv 2019, arXiv:astro-ph.IM/1910.13970.

62. Friaça, A.C.S.; Alcaniz, J.S.; Lima, J.A.S. An old quasar in a young dark energy-dominated universe? Mon. Not. R. Astron. Soc. 2005, 362, 1295-1300. [CrossRef] 